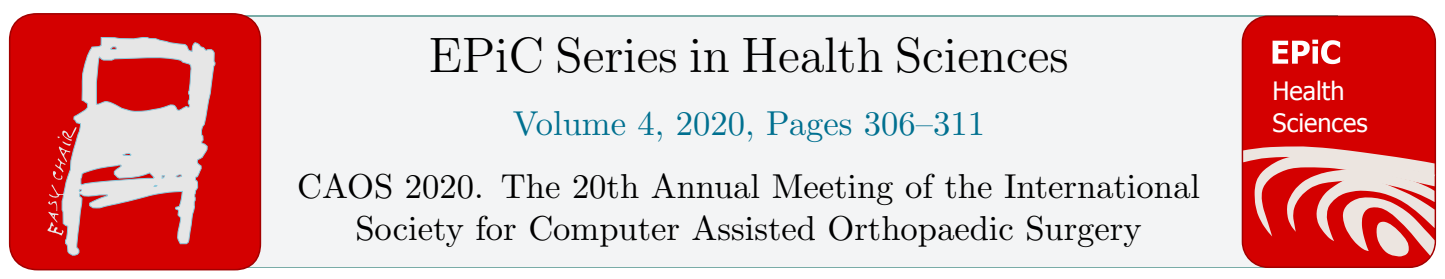

\title{
Hierarchical 3-D Registration of Computed Tomography to Ultrasound Using Reinforcement Learning Xuxin Zeng ${ }^{1}$, Michael Vives ${ }^{2}$, Ilker Hacihaliloglu ${ }^{1,3}$ \\ ${ }^{1}$ Department of Biomedical Engineering, Rutgers University, USA \\ ${ }^{2}$ Department of Orthopaedics, Rutgers New Jersey Medical School, USA \\ ${ }^{3}$ Department of Radiology, Rutgers Robert Wood Johnson Medical School, USA \\ Xuxin.zengerutgers.edu ilker.hacesoe.rutgers.edu
}

\begin{abstract}
In ultrasound (US)-based computer-assisted orthopedic surgery (CAOS), accurate and robust intra-operative registration in real-time is vital in securing the reliable outcomes for surgical image guidance. For this purpose, we focus on developing a hierarchical registration method, using reinforcement learning (RL), for 3-D registration of pre-operative computed tomography (CT) data to intra-operative US. In the RL-based registration procedure, we proposed a supervised Q-learning framework for learning the sequence of motion action to achieve the optimal alignment. Within the approach, the agent was modeled using PointNet++ with the mis-aligned point set from US and CT as the input, and the next optimal action as the output. Evaluation studies achieved average target registration error (TRE) of $3.82 \mathrm{~mm}$ with success rate of $92.7 \%$ and an average time of 8.36 seconds. We achieve $57.1 \%$ improvement in success rate over state of the art.
\end{abstract}

\section{Introduction}

Low signal to noise ratio (SNR), imaging artifacts, limited field of view (FOV) and blurred bone boundaries have hindered wide spread adaption of US in CAOS (Hacihaliloglu, 2017a). In order to provide solutions to these problems intra-operative registration methods have been investigated by various groups (Schumann, 2016). Accurate, real-time and robust intra-operative registration is of paramount importance for successful guidance in ultrasound (US)-based computer assisted orthopedic surgery (CAOS)

In (Brounstein, 2015), a Gaussian Mixture Models (GMM)-based point cloud registration method achieved a mean registration time of $2.11 \mathrm{~s}$ with a mean accuracy of $0.31 \mathrm{~mm}$. In (Pandey, 2018), an amendable and automatic image processing pipeline was proposed for CT-US registration over the full pelvis anatomy. The quantitative analysis achieved mean target registration error (TRE) of $3.22 \mathrm{~mm}$ 
and $3.89 \mathrm{~mm}$ for normalized cross-correlation (NCC) and coherent point drift (CPD) based registration methods respectively. Reported processing time for NCC-method was 25.98s (Pandey, 2018). Most recently, some studies focus on applying machine learning method for solving the registration method. In (Chen, 2016) a convolutional neural network (CNN)-based classification achieved a mean TRE of $2.3 \mathrm{~mm}$ with $82 \%$ success rate over 50 datasets on vertebras. Although these studies manifested the potential of US with the high accuracies in CT-US registration, most of the methods require a prealignment process to avoid local minima and secure accurate and robust registration outcomes.

Our main objective in this work is the development of a fully automatic, accurate and robust intraoperative registration method using reinforcement learning (RL) for US-based CAOS without the requirement for initial alignment. For that, we proposed a Q-learning based registration method (Rui Liao, 2017) with PointNet++ (Qi, 2017) based architecture to learn the optimal movement to achieve alignment. We evaluate and validate the proposed method on femur US data collected from 8 subjects.

\section{Methods}

\subsection{Data Acquisition}

The volumetric US images were collected the ultrasound simulator application package in PLUS (Lasso, 2014) and 3D slicer (Kikinis, 2014) with 500 600 images for each volume with $0.5 \sim 0.6 \mathrm{~mm}$ spacing. 10 different CT scans, obtained from 8 different subjects TCIA (Clark, 2013), were used to generate the simulated US data. Data augmentation for corresponding US-CT pairs by means of division and rotation resulted in 1600 registered US-CT cases on this dataset in total and the testing and training dataset were separately randomly with an 8:2 ratio. Training and testing data did not have scans from the same patients.

\subsection{Point Cloud Modelling from Bone Surfaces}

The first step in our proposed method is to segment to bone surfaces from the local phase information of the simulated volumetric US images we generated. The combination of local phase image features are used as an input to an L1-norm-based regularization framework for enhancement of bone shadow regions which denotes as BSE(x,y) (Hacihaliloglu, 2017a). Accordingly, simple bottom up ray casting method is used to segment the bone surfaces from the enhanced bone shadow images. With the 3-D resolution and reference position, 2D point set can be transformed into 3D point cloud which is registered to its resource models.

\subsection{Hierarchical 3-D Registration With Q-learning}

The registration learning process could be treated as an optimal strategy learning process that aims to find the optimal $4 \times 4$ transformation matrix to make the two point-based volumes aligned. Within the definition of the registration learning process, the agent is modeled using a PointNet++ based deep neural network (DNN), with the point-based data as the input and the optimal motion as the output. The proposed DNN consists of three set abstraction layers, one PointNet layer and three fully connected layers with a learning rate of 0.01 . In this proposed supervised training procedure, the agent follows a greedy registration path that always chooses the action that leads to the least distance to the target position. 


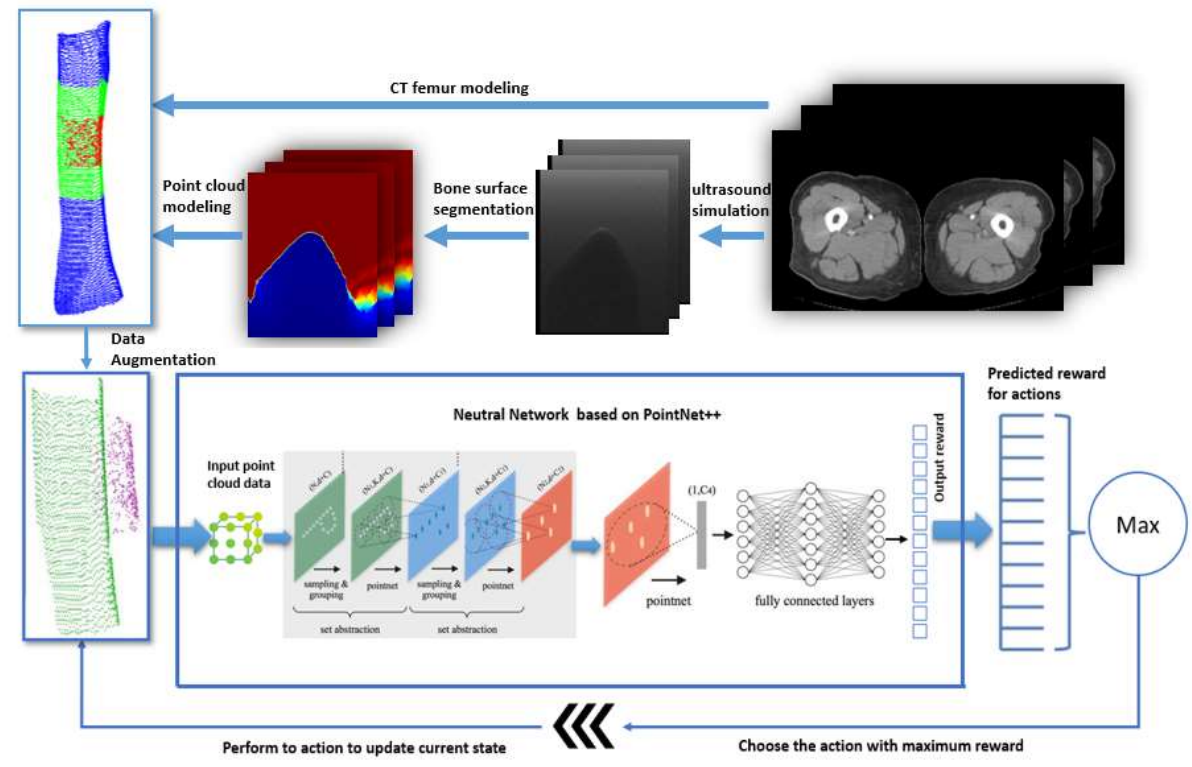

Figure 1: The framework of the proposed registration method using Q-learning, with CT data and simulated point clouds as the input and output for data acquisition, and the misalignment point clouds and the optimal action as the input and output for the Q-learning network.

\subsection{Training and Testing}

In order to increase the training data size, each aligned CT/US volumetric scan pair was randomly misaligned using rigid-body permutations within the same range of workable range for the agent, which is $\left[ \pm 20 \mathrm{~mm}, \pm 20 \mathrm{~mm}, \pm 30 \mathrm{~mm}, \pm 15^{\circ}, \pm 15^{\circ}, \pm 15^{\circ}\right]$. During training, each registered US-CT pair was augmented 3200 times by introducing random transformation during training process, resulting in more than $5 \mathrm{M}$ training data, which should be sufficient and unique enough for the reliable outcomes of the system. The training for the proposed method was conducted on a workstation with Intel Core i7 9400 @ $2.90 \mathrm{GHz}$ CPU, 16GB RAM, and Nvidia GeForce RTX 2070 Super GPU in Python 3.7. Total training time was 1 week and 2 days to converge for the proposed method. Our proposed registration method was quantitatively evaluated using target registration error (TRE), computation time, and success rate. TRE was defined as the average 3-D Euclidean distance between the anatomical landmarks extracted from both data sets (US and CT). These landmarks were not used during registration. The success rate was defined if the final calculated TRE was less than $10 \mathrm{~mm}$ (Liao R, 2017). We have also evaluated the our proposed method against three mainstream point cloud registration methods, iterative closet point (ICP) (Zhang, 1994), and coherent point drift (CPD) (Myronenko, 2009). 


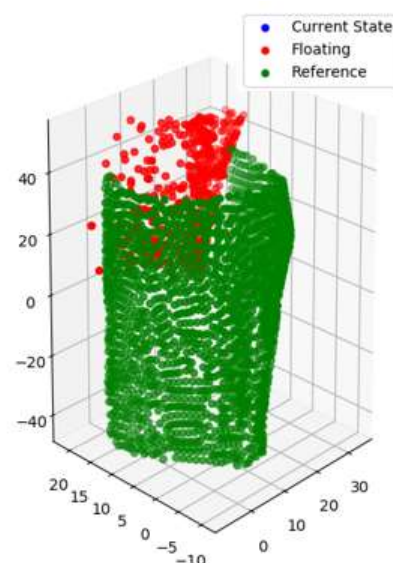

(a)

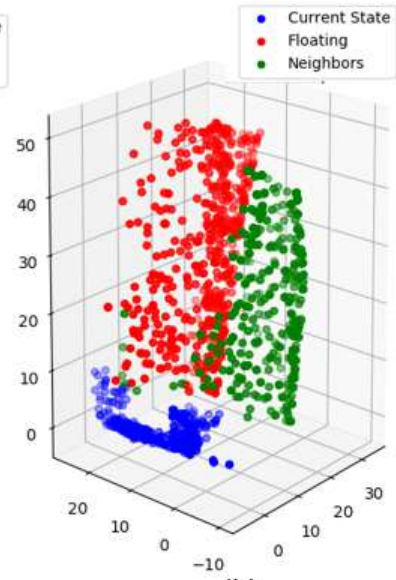

(b)

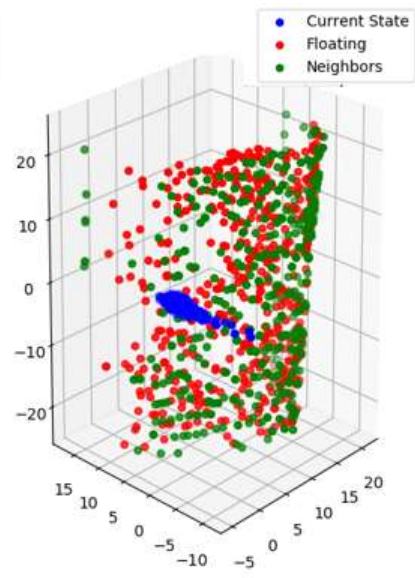

(c)

\begin{tabular}{|lccccccccc}
\hline \multicolumn{1}{c}{ TRE (mm) } & \multicolumn{3}{c}{ Time (s) } & & Success \\
\hline & Mean & Min & Max & Mean & Min & Max & 1 step & \\
\hline Proposed method & 3.82 & 2.16 & 12.42 & 8.36 & 4.75 & 18.32 & 0.31 & $92.7 \%$ \\
\hline ICP & 8.42 & 2.63 & 17.92 & 0.31 & 0.24 & 0.86 & - & $35.6 \%$ \\
CPD & 9.10 & 3.57 & 17.16 & 0.68 & 0.51 & 1.03 & - & $31.9 \%$ \\
\hline
\end{tabular}

Figure 2: (a-c) Examples of 3-D registration results using the proposed RL registration method. The images from left to right: (a) The pre-register pairs of CT and $\mathrm{KS}$ point cloud models. The green is the truncated CT model, while red is the US model. (b) The initial inputs for the RL system. The red is the US model, and the green is the neighbor points of that US model from the CT model. The input of the system, which is the difference between that models represents in blue. (c) The registration result for the mis-register pairs. (d) Registration results evaluation on the proposed method and the other point cloud registration methods. The quantitative analysis was on TRE, Time, and success rate of each registration method.

\section{Results}

The qualitative results for the proposed hierarchical registration method are shown in Fig 2 (a-c). The difference between the two objects (blue pixels) indicates the high accuracy of the proposed registration method. The quantitative results of our method are presented in Fig.2 (d). Our proposed method achieved a mean TRE of $3.82 \mathrm{~mm}(\mathrm{SD}=2.18 \mathrm{~mm})$ compared to $8.42 \mathrm{~mm}(\mathrm{SD}=3.06 \mathrm{~mm})$, and $9.10 \mathrm{~mm}(\mathrm{SD}=3.89 \mathrm{~mm})$ for ICP, and CPD methods. The mean computation time for our method was $8.36 \mathrm{~s}$ which was the most expensive one compared to the other investigated methods (Fig.2-d). We achieved 54\% and 58\% improvements for mean TRE compared to ICP and CPD methods. The success rate for the proposed method was $92.7 \%$ compared to $35.6 \%$ and $31.9 \%$ for ICP and CPD (Fig. 2-d). 


\section{Discussions and Future Work}

In this study, we proposed a 3-D rigid registration method using Q-learning which takes the pointcloud models with known transformation matrix as the inputs and outputs a continuous motion of actions to achieve the alignment between the two un-aligned models. Through the results for different lengths of volumetric US images, the proposed method demonstrated its robustness and flexibility in dealing with different registration transformations successfully without the need for initial alignment. Accordingly, we believe the proposed registration method could be used practically for medical image registration in CAOS for initial alignment of two datasets which can be further optimized using traditional point cloud-based methods. During this work we have used simulated US data which represents an easier scenario for segmentation of bone surfaces. However, for in vivo data bone surfaces could be segmented using state of the art methods such as (Alsinan AZ, 2019). Our reported mean computation time is $8.32 \mathrm{~s}$ which is not suitable for intra-operative guidance. In order to improve the computation time, we will investigate methods for sampling the point cloud data. Finally, future work will also involve the validation of the proposed method on in vivo spine scans for intra-operative registration using an US-guided CAOS system for spinal fusion surgeries.

\section{Acknowledgement}

This work was funded by North American Spine Society (NASS) 2017 young investigator basic research grant.

\section{References}

Alsinan AZVM, Hacihaliloglu IPatel. (2019). Automatic segmentation of bone surfaces from ultrasound using a filter-layer-guided CNN. International journal of computer assisted radiology and surgery, 775-783.

Anna BrounsteinIlker Hacihaliloglu, Pierre Guy, Antony Hodgson, and Rafeef Abugharbieh. "Fast and Accurate Data Extraction for Near Real-Time Registration of 3-D Ultrasound and Computed Tomography in Orthopedic Surgery." Ultrasound in Medicine \& BiologAnna,. (2015).

Chen, D. Wu, and H. Liao . "Registration of CT and Ultrasound Images of the Spine with Neural Network and Orientation Code Mutual Information." International Conference on Medical Imaging and Virtual Reality Springer International PublishingFang. (2016).

FantiFabian Torres, Eric Hazan-Lasri, Alfonso Gastelum-Strozzi, Leopoldo Ruiz-Huerta, Alberto Caballero-Ruiz, and F. Arámbula CosíoZian,. (2018). Improved Surface-Based Registration of CT and Intra-operative 3D Ultrasound of Bones. Journal of healthcare engineering .

HacihalilogluI. (2017a). Ultrasound imaging and segmentation of bone surfaces: A review. Technology, 5(02), 74-80.

Kikinis, S. D. Pieper, and K. G. Vosburgh . 3D Slicer: A Platform for Subject-Specific Image Analysis, Visualization, and Clinical Support. Intraoperative Imaging and Image-Guided Therapy. Springer New York.Ron. (2014).

Lasso, et al. "PLUS: Open-Source Toolkit for Ultrasound-Guided Intervention Systems." IEEE Transactions on Biomedical Engineering 61.10:2527-2537.Andras. (2014).

Liao RS, de Tournemire P, Grbic S, Kamen A, Mansi T, Comaniciu D.Miao. (2017). An artificial agent for robust image registration. Thirty-First AAAI Conference on Artificial Intelligence.

Myronenkoand Song, Xubo. "Point-Set Registration: Coherent Point Drift." IEEE Transactions on Pattern Analysis \& Machine Intelligence 32.12:2262-2275.Andriy,. (2009). 
Pandeyal. "Fast and automatic bone segmentation and registration of 3D ultrasound to CT for the full pelvic anatomy: a comparative study." in International Journal of Computer Assisted Radiology and Surgery.et. (2018). 1515-1524.

QiR., et al. "PointNet++: Deep Hierarchical Feature Learning on Point Sets in a Metric Space.".Charles. (2017).

Rui LiaoMiao, Pierre de Tournemire, Sasa Grbic, Ali Kamen, Tommaso Mansi, Dorin ComaniciuShun. (2017). An Artificial Agent for Robust Image Registration. in AAAI Conference.

Schumann. "State of the Art of Ultrasound-Based Registration in Computer Assisted Orthopedic Interventions." Inequality Theory \& Applications.Steffen. (2016).

Zhang. "Iterative point matching for registration of free-form curves and surfaces." International Journal of Computer Vision 13:119-152.Zhengyou. (1994). 